\title{
LARS: An Absolute Reference Spectrograph for solar observations
}

\section{Upgrade from a prototype to a turn-key system}

\author{
J. Löhner-Böttcher ${ }^{1}$, W. Schmidt ${ }^{1}$, H.-P. Doerr ${ }^{2,1}$, T. Kentischer ${ }^{1}$, T. Steinmetz ${ }^{3,4}$, R. A. Probst ${ }^{3,4}$, and R. Holzwarth ${ }^{3,4}$ \\ 1 Kiepenheuer-Institut für Sonnenphysik, Schöneckstr. 6, 79104 Freiburg, Germany \\ e-mail: jlb@leibniz-kis.de \\ 2 Max-Planck-Institut für Sonnensystemforschung, Justus-von-Liebig-Weg 3, 37077 Göttingen, Germany \\ 3 Max-Planck-Institut für Quantenoptik, Hans-Kopfermann-Strasse 1, 85748 Garching, Germany \\ 4 Menlo Systems GmbH, Am Klopferspitz 19, 82152 Martinsried, Germany
}

Received 12 May 2017 / Accepted 4 July 2017

\begin{abstract}
Context. We designed a Laser-based Absolute Reference Spectrograph (LARS) for ultra-precise solar observations. The highresolution echelle spectrograph of the Vacuum Tower Telescope is supported by a laser frequency comb to calibrate the solar spectrum on an absolute wavelength scale. In this article, we describe the scientific instrument and focus on the upgrades carried out in the last two years to turn the prototype into a turn-key system.

Aims. The goal was to improve the short-term and long-term stability of the systems, and to enable a user-friendly and more versatile operation of the instrument.

Methods. The first upgrade involved the modernization of the frequency comb. The laser system generating the comb spectrum was renewed. The Fabry-Pérot cavities were adjusted to filter to a repetition frequency of $8 \mathrm{GHz}$. A technologically matured photonic crystal fiber was implemented for spectral broadening which simplified and stabilized the setup. The new control software facilitates an automated operation of the frequency comb. The second, quite recent upgrade was performed on the optics which feed the sunlight into a single-mode fiber connected to the spectrograph. A motorized translation stage was deployed to allow the automated selection of three different fields of view with diameters of $1^{\prime \prime}, 3^{\prime \prime}$, and $10^{\prime \prime}$ for the analysis of the solar spectrum.

Results. The successful upgrades allow for long-term observations of up to several hours per day with a stable spectral accuracy of $1 \mathrm{~m} \mathrm{~s}^{-1}$ limited by the spectrograph. The instrument covers a wavelength range between $480 \mathrm{~nm}$ and $700 \mathrm{~nm}$ in the visible. Stable, user-friendly operation of the instrument is supported. The selection of the pre-aligned fiber to change the field of view can now be done within seconds.

Conclusions. LARS offers the possibility to observe absolute wavelength positions of spectral lines and Doppler velocities in the solar atmosphere. First results demonstrate the capabilities of the instrument for solar science. The accurate measurement of the solar convection, $p$-modes, and atmospheric waves will enhance our knowledge of the solar atmosphere and its physical conditions to improve current atmospheric models.
\end{abstract}

Key words. instrumentation: spectrographs - instrumentation: miscellaneous - Sun: photosphere - line: profiles

\section{Introduction}

Observational solar physics relies strongly on precise and accurate spectroscopy. The optical solar spectrum with its thousands of spectral lines provides a wealth of information about material motions in the light-emitting layers of the Sun, the presence of magnetic fields, but also gas temperature, ionization state, and other factors. The Sun allows us to measure spectral line profiles with high spectral and spatial resolution in order to investigate the conditions in the solar atmosphere in three dimensions. Asymmetric line profiles provide information about gradients of physical quantities with height in the solar atmosphere, for example near-surface convection, acoustic waves, or magnetic reconnection. Moreover, due to the solar activity, the conditions can vary at a temporal scale of seconds to hours and they also depend on the heliocentric angle on the solar disk.

The investigation of motions and other physical conditions in the solar atmosphere thus requires the precise measurement of the spectral line profiles and positions. A high temporal cadence, a well-defined averaging area, and a distinct repeatability for the measurements are imperative apart from the high spectral resolution. With existing grating spectrographs and filter spectrometers, the typical wavelength accuracy amounts to around $2 \mathrm{~m} \AA\left(\sim 100 \mathrm{~m} \mathrm{~s}^{-1}\right)$ in the visible range. Former techniques to calibrate the wavelengths of the solar spectrum are the use of iodine cells (Beckers 1977), spectral lamps (Lovis \& Pepe 2007), a Fabry-Pérot interferometer (Reiners et al. 2014), or telluric lines as reference. However, the application of all given methods is limited, either by the low intensity level of the reference lines, their irregular distribution across the spectral range, or their known accuracy. The Laser Absolute Reference Spectrograph (LARS) overcomes all of these issues by using a laser frequency comb (LFC) to calibrate the solar spectrum recorded with a highresolution echelle spectrograph, thus accomplishing a consistent accuracy better by two orders of magnitude. For solar physics, this makes LARS a unique instrument.

In astrophysical spectroscopy, a high spectral accuracy is imperative to reliably measure small-scale velocities of the gas at the solar surface. All systematic effects (like orbital motions, rotations, and gravitational shifts) on the solar spectrum need to be 


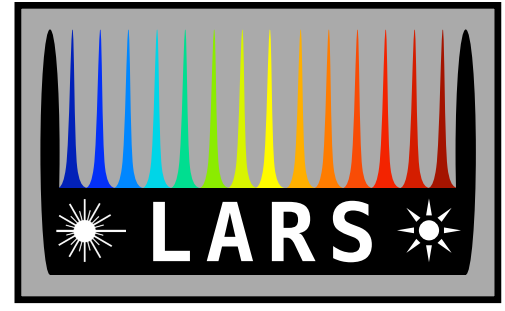

Fig. 1. Logo of the LARS instrument. The emission lines of the laser resemble the shape of a comb. The colors from blue to red indicate the operating range of the instrument in the visible. The symbols refer to the assembly of laser and solar spectroscopy.

known or measured with sufficient accuracy to guarantee the best quality data for a careful and consistent data calibration. In addition, for many astrophysical investigations a high short-term and long-term stability is essential. To investigate large-scale flows, or global properties, like the convective blueshift, long averaging times are needed to eliminate small-scale convective and oscillatory motions that act as unwanted "solar noise". With a highresolution telescope like the German Vacuum Tower Telescope (VTT, Schroeter et al. 1985) which observes only a small field of view, the significant systematic measurement of, for example, the center-to-limb variation of the convective blueshift may take weeks to months. Sensitive and very stable instruments are required to avoid the detrimental influence of unknown drifts, caused, for example, by changes in the index of refraction of the ambient air. At the VTT, these issues are solved with the Laser Absolute Reference Spectrograph (see logo in Fig. 1), a system based on an LFC as an absolute calibration source. LARS provides an accurate wavelength calibration of each measurement over a large continuous wavelength range, and it guarantees long-term consistency of spectroscopic observations over months and years.

In a pioneering work, Steinmetz et al. (2008) demonstrated the feasibility of calibrating an astronomical spectrograph with a laser frequency comb. The authors used a comb operated in the infrared range to successfully calibrate the echelle spectrograph of the VTT. Between 2010 and 2013, an LFC-based wavelength calibration system was developed for the VTT spectrograph in a cooperation between the Kiepenheuer Institute for Solar Physics, Freiburg, the Max Planck Institute of Quantum Optics, Garching, and Menlo Systems GmbH, Martinsried. Initially, the system was planned to cover the visible spectral range from $500 \mathrm{~nm}$ to $600 \mathrm{~nm}$ with a mode separation of about $5 \mathrm{pm}$. Due to the high spectral resolution of the VTT spectrograph, the chosen mode separation was considered to be narrow enough to have a sufficient number of comb modes as calibration lines everywhere in the spectrum. At the same time, the separation was large enough to observe clearly distinguishable comb lines with the shape of the spectrograph's points spread function (Doerr et al. 2012b; Doerr 2015; Probst et al. 2015).

This paper describes the LARS instrument in its current science-ready configuration that was reached after substantial upgrades and modifications in 2016 and 2017, compared to the prototype version described in earlier works (Doerr et al. 2012a,b; Doerr 2015; Probst et al. 2015). In Sect. 2, we briefly touch on the properties of the telescope and spectrograph, followed by a description of the final setup of the LFC that now allows for turn-key operation of the instrument. The final part (Sect. 2.4) deals with the new opto-mechanical interface between the telescope and the single-mode fiber feed with fields of view between $1^{\prime \prime}$ and $10^{\prime \prime}$ in diameter. In Sect. 3, we demonstrate the

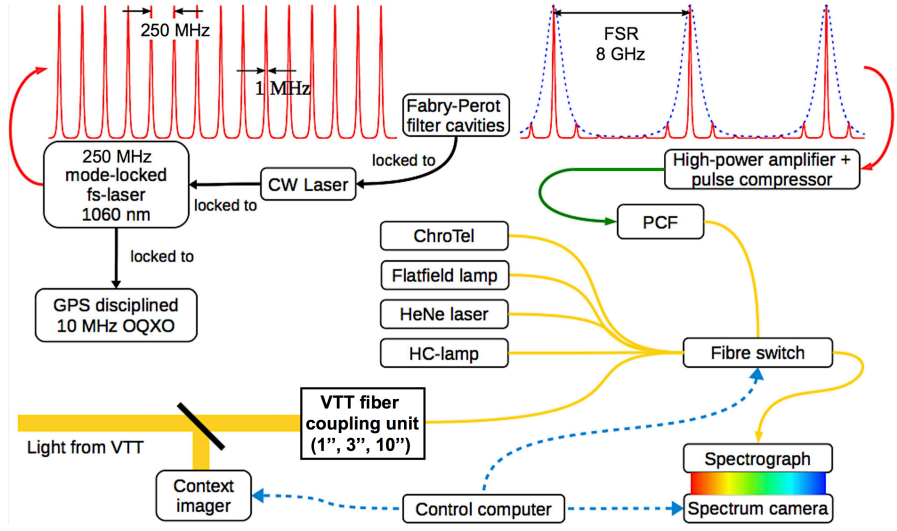

Fig. 2. LARS system. The femtosecond ytterbium laser operates at a wavelength of $1060 \mathrm{~nm}$ with a repetition rate of $250 \mathrm{MHz}$, and is locked to a GPS-disciplined oscillator. The generated emission spectrum (upper left) passes a pair of identical Fabry-Pérot cavities which filter the modes to an output repetition rate of $8 \mathrm{GHz}$ (upper right). The frequency comb spectrum is amplified and then broadened by a photonic crystal fiber (PCF). With single-mode fibers (yellow lines), the signal from each light source (LFC, sunlight, flatfield lamp, HeNe laser, hollowcathode lamp, ChroTel) is guided to its own entrance port at the fiber switch. One output port is connected to the spectrograph (lower right). The light from the VTT falls onto a beamsplitter (lower left) and enters a fiber-coupling unit $\left(1^{\prime \prime}, 3^{\prime \prime}\right.$, or $\left.10^{\prime \prime}\right)$ for spectral measurements and a camera for context imaging of the surrounding solar region. Acronyms are explained in Sects. 2.1 to 2.3. Figure adapted from Doerr (2015).

scientific performance of the instrument. Section 4 provides an outlook to the observing programs planned with LARS and the scientific opportunities offered by the instrument.

Throughout the paper we adopt common practice in astrophysics and use air wavelengths when referring to spectral lines or observed wavelengths. The difference to vacuum wavelength (which is linked to frequency $v$ and speed of light $c$ through the well-known relation $\lambda \cdot v=c$ ) is about $0.14 \mathrm{~nm}$ in the range around $500 \mathrm{~nm}$. An equation derived by Edlén $(1953,1966)$ is used to convert vacuum to air wavelengths, or vice versa.

\section{Instrumental setup}

In this section, we describe the instrument in its final scienceready configuration which was reached after a substantial upgrade of the frequency comb in 2016 and of the optical setup of the solar light channel in 2017.

\subsection{LARS system}

LARS combines a number of optical subsystems in one powerful wavelength-calibrated solar spectrograph. A short overview of the full instrumental setup and the involved subsystems is given in this section. The schematic overview of the setup is shown in Fig. 2. The telescope and spectrograph are described in Sect. 2.2. Within the scope of the instrumental upgrades, the laser frequency comb and the new fiber-coupling of the solar light will be explained in more detail in Sects. 2.3 and 2.4.

LARS was developed to perform solar observations supported by an LFC as a source of frequency (or wavelength) calibration. The conjunction of both elements is illustrated in Fig. 2. The upper part sketches the generation of the frequency comb, the lower part shows the fiber-coupling of the solar light to the spectrograph. The sunlight collected by the VTT falls onto a cubic beam splitter which reflects $10 \%$ of the incoming light 
to a camera imaging the solar region as it appears in a selected narrow wavelength range. The passing $90 \%$ of the sunlight is guided to a fiber-coupling unit by which the light of a selected field aperture $\left(1^{\prime \prime}, 3^{\prime \prime}\right.$, or $10^{\prime \prime}$ on the solar disk) is fed into a single-mode fiber. The integrated signal is then guided to a fiber switch which can rapidly change between eight entrance ports. The single output port is connected to the spectrograph for spectral observations.

As depicted in Fig. 2, there are five different other light sources connected with fibers to the switch: i) A tungsten lamp which is used as a fiber-coupled flatfield lamp producing a continuous white-light spectrum for the calibration of the spectrograph camera; ii) a stabilized HeNe laser producing a sharp emission line to optimize the spectrograph alignment; iii) several hollow-cathode lamps which can be employed to measure the spectral emission of their atomic transitions; iv) the integrated full-disk light from the Chromospheric Telescope (ChroTel, Kentischer et al. 2008); v) the laser frequency comb to absolutely calibrate the spectrograph and wavelength scale of all other spectra.

\subsection{Telescope and spectrograph}

For the sake of completeness, we briefly summarize the main properties of the VTT and its echelle spectrograph. Parts of the setup are sketched in the bottom of the setup overview in Fig. 2. More information can be found on the VTT webpage ${ }^{1}$.

The $70 \mathrm{~cm}$ telescope has a focal length of $45 \mathrm{~m}$ and images a circular fraction of the solar disk $\left(270^{\prime \prime} \varnothing\right)$ at the main focal plane, with a plate scale of $4.5^{\prime \prime} \mathrm{mm}^{-1}$. The entrance slit of the spectrograph is located at the focal plane in the direct, vertical beam. A $45^{\circ}$ fold mirror allows us to redirect the beam to various optical laboratories. For observations with LARS, the entrance slit is replaced by a fiber-coupling unit. A single-mode fiber (SMF) with a mode-field diameter of a few $\mu$ m feeds the light (solar or artificial) to the spectrograph. This guarantees an identical illumination of the spectrograph for all light sources. The spectrograph design matches the telescope's ratio between focal length and aperture of 64, whereas the numerical aperture of the SMF corresponds to an f-ratio of 3.8. To adapt the SMF to the spectrograph, an asphere with a short focal length is mounted between the fiber and the entrance focal plane to generate a beam with an f-ratio of about 64 that illuminates the large 79-grooves-per-inch echelle grating. We note that the spectrograph is not a classical, cross-dispersed echelle instrument. Instead, a pre-disperser produces a spectrum with low dispersion (about $1.6 \mathrm{~nm} \mathrm{~mm}^{-1}$ ) at the entrance focal plane of the main disperser. The main part of the spectrograph is an asymmetric allreflective Czerny-Turner instrument with a $15 \mathrm{~m}$ collimator and a $7.5 \mathrm{~m}$ imaging mirror. This leads to a demagnification of the spatial scale by a factor of two compared to the telescope and to a dispersion of $19 \mathrm{pm} \mathrm{mm}^{-1}(\lambda=550 \mathrm{~nm})$. The spectral resolution $R=\Delta \lambda / \lambda$ amounts to about 800000 at a wavelength of $550 \mathrm{~nm}$. To record the spectra, we use an ANDOR NEWTON camera with a charge-coupled device (CCD) chip with a pixel size of $13.5 \mu \mathrm{m}$ and a chip size of $2048 \times 512$ pixels. This results in a spectral field of view of $0.53 \mathrm{~nm}$ at a wavelength of $550 \mathrm{~nm}$ (Doerr 2015).

\footnotetext{
1 http://www. leibniz-kis.de/en/observatories/vtt/ vtt-instrumentation/
}

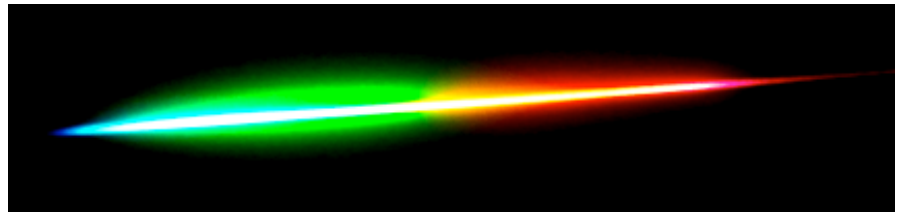

Fig. 3. Broadened spectrum of LARS. For illustration, the light leaving the optical fiber was dispersed with a small diffraction grating and projected onto a nearby wall. Toward the red, the wavelength range is limited by the transmission range of the single-mode fiber (and by the sensitivity of the camera used for the photograph). The blue end depends on the optical power used for spectral broadening of the frequency comb. In this example we reach a wavelength coverage of about $480 \mathrm{~nm}$ to $700 \mathrm{~nm}$.

\subsection{Key upgrade 1: the frequency comb}

The major step from the expert-user prototype version of LARS to a turn-key systems was taken in May 2016. The upgrade of the LFC now enables a user-friendly long-term stable operation of the instrument. The setup and its key upgrade are described in the following.

A femtosecond laser operating at a center wavelength of $1060 \mathrm{~nm}$ generates a comb of equally spaced modes with a mode separation of $250 \mathrm{MHz}$. The native comb spectrum is sketched in the upper left of Fig. 2. The laser itself is referenced to a commercial $10 \mathrm{MHz}$ oven-controlled quartz crystal oscillator (OQXO), that is GPS-disciplined. To convert a frequency comb to an instrument suitable for astrophysical applications ("astro-comb"), the repetition rate and the spectral band of the laser have to be adapted to the capabilities of the spectrograph under consideration. This is achieved by sending the laser light through a pair of stabilized Fabry-Pérot cavities, which transmit only every 32nd comb mode. The cavities are locked to the repetition rate of the LFC by the transmission signal of a continuous wave (CW) laser. The resulting output signal is sketched in the top right corner of Fig. 2. The output repetition rate (mode separation) amounts to $8.0 \mathrm{GHz}$. The signal is amplified in a cladding-pumped $\mathrm{Yb}$ doped fiber amplifier, and thereafter broadened in a tapered photonic crystal fiber (PCF). The final, spectrally broadened LFC signal is shown in Fig. 3 with low dispersion. The light exiting the PCF is guided through a single-mode fiber to an additional free-space unit with a knife-edge (not depicted in Fig. 2). By blocking parts of the light beam, the intensity level of the comb light is adjusted to the continuum level of the solar spectrum. The regulated comb signal is coupled to another singlemode fiber and guided to the fiber switch. The general design of an astro-comb has already been described by Steinmetz et al. (2008) and Wilken et al. (2012). Doerr (2015) and Probst et al. (2015) discussed the prototype version of the present instrument in great detail.

In the prototype version (Doerr 2015), the infrared laser signal was translated into the visible range by using a second harmonic generator, which doubled the frequency. An earlier version of the tapered PCF then broadened the laser signal to a range from $460 \mathrm{~nm}$ to $700 \mathrm{~nm}$. In the meantime, the PCF technique matured by optimizing the taper geometry and PCF structure. The tapered elliptical core of the PCF spans only a few micrometers. The substantially wider broadening of the fundamental comb spectrum makes the use of a second-harmonic generator obsolete. The frequency comb spectrum now spans from $480 \mathrm{~nm}$ to $1300 \mathrm{~nm}$. This was a major step forward, simplifying the optical setup and, at the same time, substantially increasing the operational stability. The new PCFs not only provide 


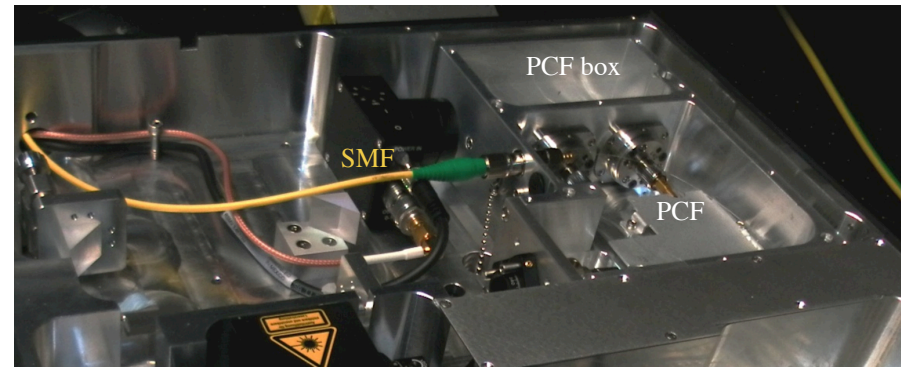

Fig. 4. Photonic crystal fiber (PCF) is connectorized at both ends, with sealed end facets, and is mounted in a segregated housing (box in the right part of the picture) for quick and easy replacement. The PCF output is coupled to a single-mode fiber (yellow) and guided to the knifeedge unit and fiber switch.

much larger spectral broadening, they also have a significantly longer life time of several thousands of operating hours. They are readily available as plug-and-replace units which can be exchanged quickly. The PCF and its box are shown in Fig. 4. As a by-product of this development, all optics are now fully contained in optical enclosures, which again improved the stability and decreased the sensitivity to changes in the ambient conditions. The new software package controls all instruments' parameters and regulates them automatically to the optimized setting. Eight weeks of observation time in 2016 have verified an uninterrupted system stability of up to several days.

In the prototype version, the native laser mode separation of $247.5 \mathrm{MHz}$ had been filtered to $5.445 \mathrm{GHz}$. This number translated into a mode separation of $4.5 \mathrm{pm}$ (at $\lambda=500 \mathrm{~nm}$ ), well resolved by the spectrograph. On the other hand, the mode spacing was large enough to clearly identify the wavelength of an individual laser mode due to its proximity to a solar spectral line. Once the wavelength (and thus the frequency) of a single laser mode is identified, all other modes are exactly known, due to the given offset frequency and the constant spacing of the frequency modes. For the final setup we decided to change the mode separation from $5.445 \mathrm{GHz}$ to $8.0 \mathrm{GHz}$. Thus, only every $32 \mathrm{nd}$ mode is transmitted by the Fabry-Pérot cavities. The mode separation of $8.0 \mathrm{GHz}(6.67 \mathrm{pm}$ at $\lambda=500 \mathrm{~nm})$ is still convenient for the visible wavelength range and for the VTT spectrograph with its extremely high spectral resolution. In addition, the larger repetition rate would enable the use of LARS also for the GREGOR Infrared Spectrograph (GRIS, Collados et al. 2012) at the GREGOR solar telescope (Schmidt et al. 2012).

Intrinsically, the LFC provides a wavelength accuracy at the level of a few $\mathrm{cm} \mathrm{s}^{-1}$. The stability of the GPS-disciplined reference oscillator ultimately limits the frequency accuracy of the comb to an absolute value of only $3 \mathrm{~mm} \mathrm{~s}^{-1}$. It is the spectral filtering of the native comb with the Fabry-Pérot cavities (FPC) which introduces a minimal mismatch between the FPC transmission peaks and the equidistant comb peaks. As a result, the side-modes are suppressed with a slightly asymmetric weight. Then, fitting the transmitted modes with a Gaussian leads to a worst-case shift of $2.1 \mathrm{~cm} \mathrm{~s}^{-1}$. In addition, during the power amplification for spectral broadening, the side-modes are unavoidably re-amplified to an intensity of around $-67 \mathrm{~dB}$ relative to the transmitted mode. This imperfection is tremendously small compared to other effects (Wilken et al. 2012), but introduces an additional defective shift of the LFC line centroids of $5 \mathrm{kHz}$, or $3 \mathrm{~mm} \mathrm{~s}^{-1}$ at $630 \mathrm{~nm}$ (Doerr 2015).

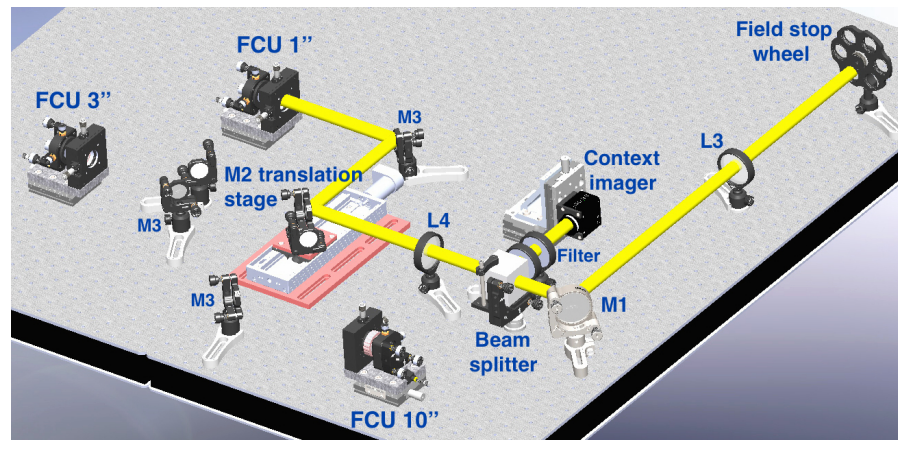

Fig. 5. New optical setup for solar observations with LARS. The fibercoupling units (FCU), lenses (L), mirrors (M), translation stage, and Context Imager are sketched.

\subsection{Key upgrade 2: fiber-coupling of the solar light to the spectrograph}

The second major upgrade of LARS was performed in April 2017. The optical setup of the solar channel was restructured to facilitate a quick change between three available field apertures for solar observations. On this occasion, the majority of the optical components were replaced.

As illustrated in Fig. 2, LARS observes the solar light simultaneously with two cameras. The Context Imager records a twodimensional image of the solar region captured by the telescope. At the same time, a small circular field in the center of that region is fed into a single-mode fiber and its field-integrated spectrum is observed by the spectrograph camera. Three fiber-coupling units are available to measure the sunlight, with an aperture corresponding to either $1^{\prime \prime}, 3^{\prime \prime}$, or $10^{\prime \prime}$ on the sky. For solar observations, such a distinction of the integrated region is necessary to perform exclusive and qualitative measurements of the atmospheric conditions for different spatially-resolved solar features, for example, solar granules with a size of $1^{\prime \prime}$ or umbrae with a diameter of $10^{\prime \prime}$. A pinhole in the final focus limits the field and a collimating lens with short focal length feeds the light to the fiber facet. Since it is not possible to image the observed aperture on the tiny fibre core, we project the telescope pupil on the fibre facet. This ensures that information from the whole aperture is coupled to the fibre. But owing to the Gaussian acceptance profile of the fibre, the inner regions of the aperture are covered with higher efficiency than the outer regions. Nevertheless, the optics were designed such that the coupling efficiency in the outer regions of the aperture does not drop below $60 \%$. During observations, a change of the fiber-coupled aperture involved a manual exchange of the pinhole and lens in front of the fiber. Since this change included a sensitive optical alignment to maximize the coupling efficiency, this process was laborious and time-consuming. Therefore, the basic idea of the upgrade was to install three individual pre-aligned fiber-coupling units, one for each field aperture. A motorized translation stage with two mounted silver-coated mirrors guides the light to one of the fiber-coupling units. This automatization now enables a selection of the different apertures within seconds.

The old setup (Doerr 2015) consisted of an X95 rail and carrier system to align the optical components with the light path. As part of the upgrade, the rail system was replaced by two $60 \mathrm{~mm}$ optical breadboards for the new optics. The inner honeycomb structure optimizes the damping of vibrations and acoustic disturbances below $100 \mathrm{~Hz}$. The new optical design is displayed in Fig. 5. The transfer optics consisting of two lenses (L1 and L2, not shown here) with focal lengths of $1500 \mathrm{~mm}$ relay the 


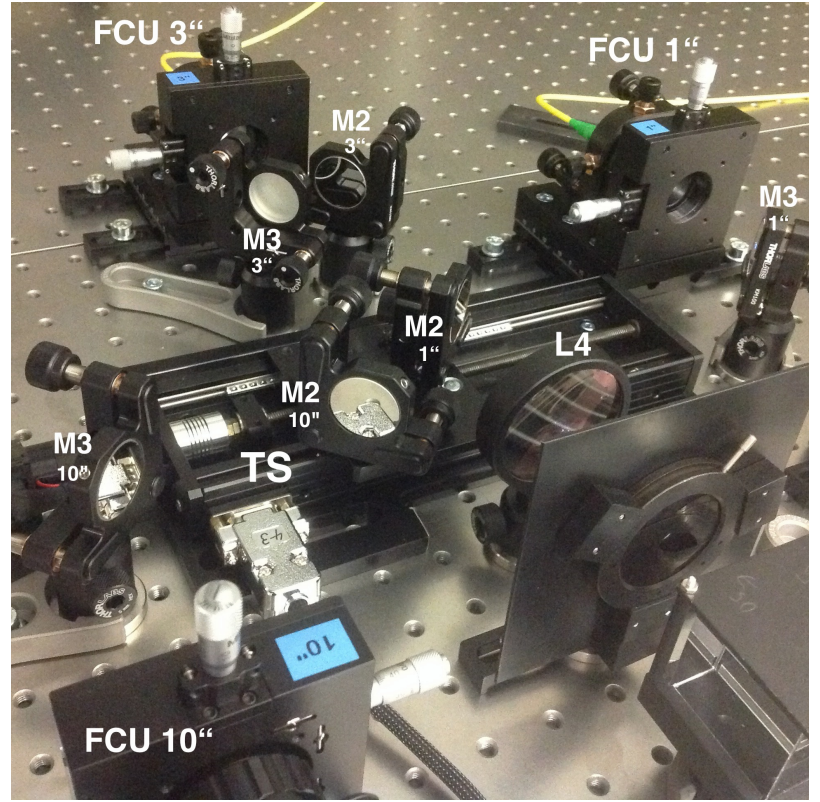

Fig. 6. Fiber-coupling units. The solar light of the respective field of view $\left(1^{\prime \prime}, 3^{\prime \prime}\right.$, or $\left.10^{\prime \prime}\right)$ is fed to the fibers. The integrated signal is guided to the spectrograph. Compare Fig. 5.

science focus of the VTT toward a stop wheel with a set of removable blends. Besides the free field, three pinholes with different field apertures ( $1^{\prime \prime}, 3^{\prime \prime}, 10^{\prime \prime}$ on the sky) are mounted at the wheel to align the light path to the fiber and context camera. A third $50 \mathrm{~mm}$ diameter achromatic lens (L3) with a focal length of $300 \mathrm{~mm}$ collimates the light to the silver-coated mirror (M1). The high-precision two-axes adjusters of the mirror mount facilitate the optimized alignment of the light beam. After the mirror, a cubic beamsplitter reflects $10 \%$ of the incoming light to the Context Imager. A combination of a $50 \mathrm{~mm}$ lens and an interference filter focus the wavelength-filtered light onto the CCD chip of the Context Imager camera. Information about the data acquisition is given in Sect. 3.1.

The remaining $90 \%$ of the light passes the beamsplitter toward the fiber-coupling units (see Fig. 6). A $50 \mathrm{~mm}$ achromatic lens (L4) with a focal length of $300 \mathrm{~mm}$ focuses the light toward the pinhole of the fiber-coupling unit. The heart of the system upgrade is the increase from one to three individual fiber-coupling units. A motorized translation stage (TS) manufactured at the Kiepenheuer-Institut handles the beam guiding. Two silver mirrors (M2) with diameters of $25.4 \mathrm{~mm}$ are mounted on the moveable stage with an angle of incidence of $\pm 45^{\circ}$ to the incoming light beam. Three pre-adjusted positions (first, second, or no mirror) can be selected with micrometer repeatability. In combination with an additional folding mirror (M3), an identical length of the light path to all fiber-coupling units is guaranteed. All mirrors are mounted in two-axis adjusters to enable a precise and stable alignment.

The fiber-coupling unit feeds the sunlight into the optical single-mode fiber. The optical components are displayed in Fig. 7. A circular pinhole is drilled into a brass plate to limit the field aperture to $1^{\prime \prime}, 3^{\prime \prime}$, or $10^{\prime \prime}$ on the solar disk. The lens with short focal length collimates the residual light beam toward the fiber facet. To maximize the coupling efficiency into the fiber, the fiber-coupling unit in total features nine degrees of freedom for orientation and alignment. The whole unit is placed on top of a dovetail translation stage which can shift the unit by $25 \mathrm{~mm}$ in the direction of the light path. The pinhole and lens can be

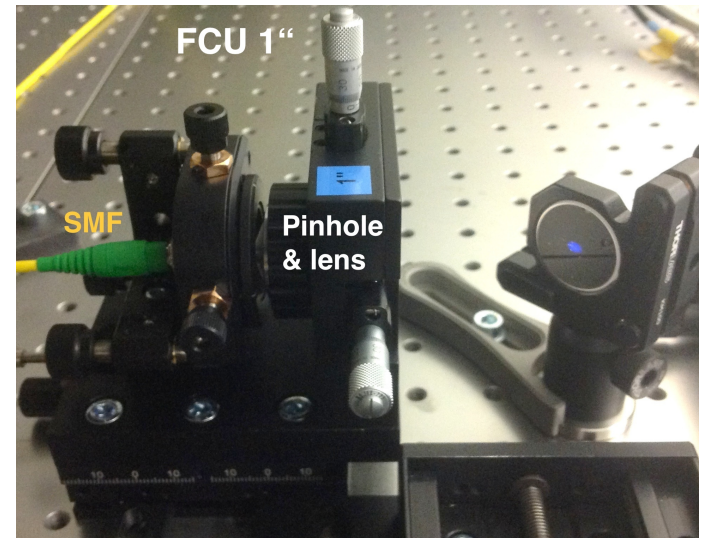

Fig. 7. Fiber-coupling unit with pinhole, lens, and single-mode fiber (SMF) of the $1^{\prime \prime}$ field.

moved along two axes, vertically and horizontally, with respect to the fiber. The fiber mount itself can be adjusted along six axes. To bring the fiber into the optimal position along the direction of the light beam, the unit is placed on top of a one-axis translator. The fiber head itself has a five-axes mount for optical alignment in the $X, Y$, and $Z$ direction, as well as tip and tilt. The light coupled to the fiber (yellow cable in Fig. 7) is guided internally to the fiber switch. When the VTT channel is selected by the control software, the light enters the output fiber which is connected to the spectrograph.

All optical fibers of LARS are single-mode fibers. The fibers are specified for a peak transmission in the range from $450 \mathrm{~nm}$ up to $700 \mathrm{~nm}$. Due to the operating range of the frequency comb starting at $480 \mathrm{~nm}$, this limits LARS observations to the visible part of the spectrum from $480 \mathrm{~nm}$ to $700 \mathrm{~nm}$. Toward the red, the damping due to the fiber necessitates exposure times of a few seconds to perform spectral observations. Unlike multi-mode fibers, single-mode fibers guide only one propagation mode. This brings the advantage of well-defined beam properties which are completely independent of the input coupling parameters. A stable and uniform illumination of the spectrograph grating is achieved, which in turn enables the high calibration accuracy of the spectra. On the other hand, the coupling efficiency of the incoming light is very low. But since the Sun (compared to other stars) is an extended source with high intensity, the light level transmitted through the single-mode fiber is always adequate to allow for high-cadence $(\sim 1 \mathrm{~Hz})$ solar spectroscopy.

\subsection{Instrument control}

For spectroscopic observations with LARS, a number of independent control units are involved. A schematic overview is given in Fig. 8. It contains: (i) the telescope operation system and pointing, as well as the adaptive optics (AO) system; (ii) the spectrograph with its echelle grating and predisperser; (iii) the different units operated by the LARS control software (these include the spectrograph CCD camera, the translation stage selecting the active fiber-coupling unit, the fiber switch to select the input source for the spectrograph, the Context Imager, and a knife-edge unit to regulate the light level of the frequency comb); and (iv) the frequency comb itself with its operation control and monitoring. The four main units are operated independently by the observer. The following sections describe the last two units. 


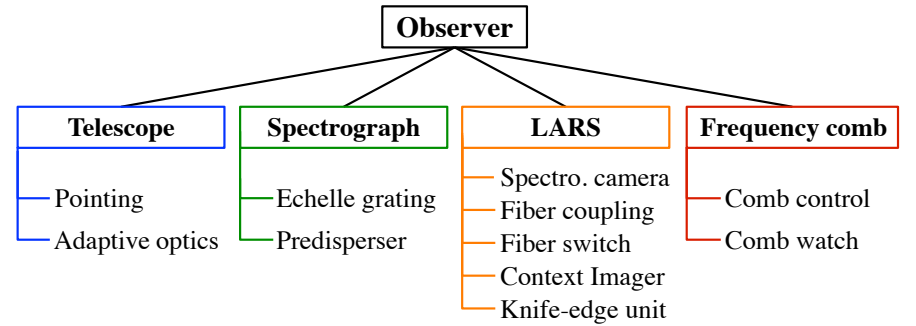

Fig. 8. Schematic overview of the instrument control. The observer operates four individual control units and their sub-units.

\subsubsection{LARS control}

The LARS control software commands the five sub-units listed in Fig. 8 (third column from the left). It sets the observational parameters for the spectrograph camera, like the integration time of the CCD, the read-out area and binning mode, the temporal cadence and number of repetitions within the sequence, and the camera cooling. It also controls the fiber switch to alternate between the selected input channels of the different light sources. The Context Imager can be operated individually or triggered by the spectrograph camera. The knife-edge unit is driven to regulate the light level of the frequency comb. The control software package was supplemented by the automated translation of the fiber-coupling units for the field of view. Before the optical upgrade described in Sect. 2.4, the change of the fiber-coupling unit had to be performed by hand, including optical alignment to maximize the coupling efficiency. This was laborious and timeconsuming. The new motorized translation stage and pre-aligned fiber-coupling units allow an automated selection of the different fields of view within a few seconds with a high repeatability. Its operation is now included in the LARS control software as part of the upgrade.

\subsubsection{Frequency comb control}

Although it is one of several light sources connected to the fiber switch, the LFC and its control system are operated independently from the rest of the instrument (see right column in Fig. 8). The new version of the comb control system that came with the upgrade in 2016 (see Sect. 2.3) now allows for an automated operation of the comb. This was the decisive step to convert the whole system from a complex prototype into a (still complex, but easily manageable) turn-key instrument. The LFC control system can be operated remotely, for example, from the VTT control room, or from any authorized computer outside the observatory. This remote capability allows for remote support by experts in case of a malfunction. To check or reinspect the correct operation of the LFC system, an internal log file with all context information is written.

\subsubsection{Remote control station}

To facilitate solar observations with LARS, all required control screens can be bundled as remote sessions at the remote control station (see Fig. 9) in the control room of the VTT. Experienced users are thus able to perform LARS observations singlehandedly. All remote sessions can also be controlled from any other authorized computer.

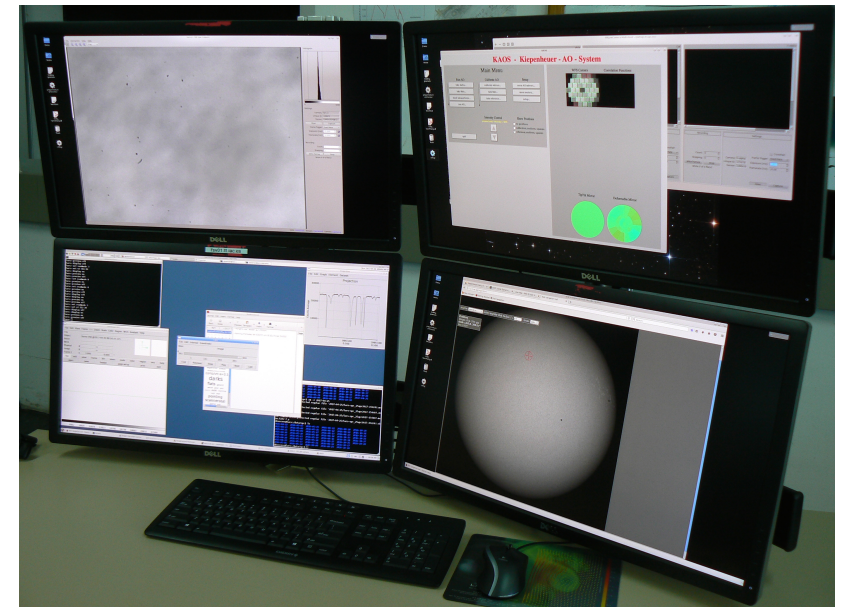

Fig. 9. Remote control station at the VTT. All instruments and devices needed for LARS observations are accessible via remote desktop tools and are displayed on an array of screens.

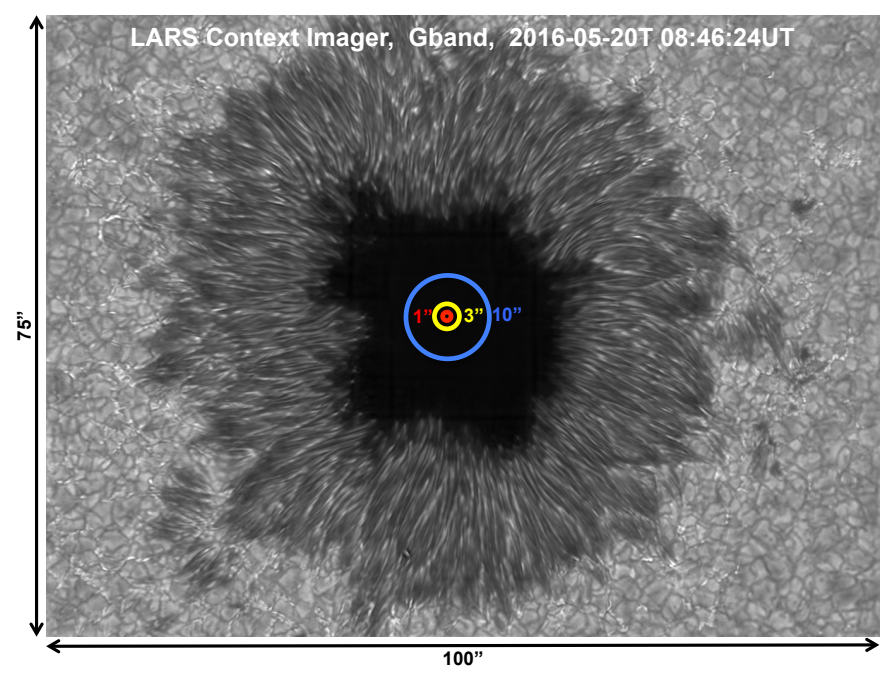

Fig. 10. Sunspot observed with the Context Imager of LARS. The image sequence was recorded on May 202016 at 08:46 UT with an interference filter centered on the spectral $G$-band around $430 \mathrm{~nm}$. The Speckle-code KISIP was used to reconstruct the displayed image. The full field of view covers a size of $100^{\prime \prime} \times 75^{\prime \prime}$. The light from a circular region with a diameter of either $1^{\prime \prime}$ (red), $3^{\prime \prime}$ (yellow), or 10" (blue) is integrated to a fiber and guided to the spectrograph.

\section{Observations and data processing}

In this section, we discuss the observations which are performed with the two cameras of LARS - the Context Imager (Sect. 3.1) and the spectrograph camera (Sect. 3.2). We thereby focus on the acquired data and give a summary of the data calibration for both channels.

\subsection{Context Imager}

Observation: the Context Imager records a two-dimensional image of the Sun. The sunlight is filtered to one narrow wavelength range of typically $1 \mathrm{~nm}$ or less. Various spectral pre-filters can be inserted to yield context information, for example, about photospheric, chromospheric, or magnetic dynamics in the solar atmosphere. An example of a recorded context image is displayed in Fig. 10. It shows a fully-developed sunspot recorded 


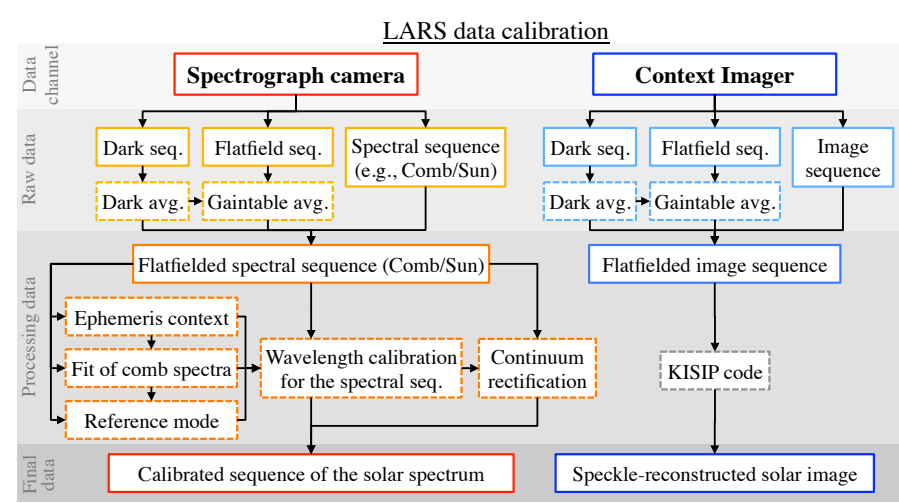

Fig. 11. Calibration scheme for LARS data. The data reduction from the raw to the final state is displayed for both LARS channels - the spectrograph camera (red colors) and the Context Imager (blue colors). Black arrows indicate the direction of processing. Raw, processing, and final state products are drawn with solid borders around the text box. Intermediate calibration steps and tools are indicated by dashed borders

on May 202016 at 08:46 UT close to the solar disk center at a wavelength of $430.8 \mathrm{~nm}$ ( $G$-band). The reasons for selecting this spectral band were the simplified distinction between magnetic and non-magnetic regions, and the naturally higher spatial resolution in the blue. With the appropriate pre-filter, the Context Imager can be operated at any wavelength in the visible. The CCD camera has a detector size of $1360 \times 1024$ pixel, translated into a full field of view of $100^{\prime \prime} \times 75^{\prime \prime}$ on the Sun. The Context Imager can be synchronized to the spectrograph camera. Then, for every acquired spectrum, a context image is recorded simultaneously. The Context Imager can also be operated independently. Sequences with up to 20 frames per second and exposure times of a few milliseconds can be achieved. Adaptive optics can be enabled to correct for image distortions caused by atmospheric turbulences. Image reconstruction techniques are then applied to reach a spatial resolution close to the diffraction limit of the telescope. The two-dimensional image serves as context information for the spectroscopic observation. The three different fields of view and their alignment with respect to the context image are indicated in Fig. 10 as red $\left(1^{\prime \prime}\right)$, yellow $\left(3^{\prime \prime}\right)$, and blue $\left(10^{\prime \prime}\right)$ circles.

Data calibration: the raw data recorded with the Context Imager contain several defects like dissimilar pixel sensitivities, dust or dirt on the detector, and atmospheric distortions of the solar image which are corrected by the data calibration. The set of calibration data includes a sequence of "darks" to evaluate the background signal. A sequence of "flatfields" (smeared whitelight images by performing a fast movement over a Quiet Sun region) is recorded to determine intensity defects, and to calculate the average gaintable image for the flatfield-correction of the image sequence. The calibration scheme for the Context Imager data is shown on the right side of Fig. 11. As a matter of routine, a set of context images is recorded (not named in the processing scheme). An AO-stabilized image of a US Air Force test target is taken to gain information on the spatial resolution. A pinhole image is obtained to verify the position of the AO lock point. Finally, context information on the location of the fiber and the size of the integrated region is taken for the spectral observation. The respective field stop at the filter wheel is inserted and a field-of-view image of the fiber is recorded. The flatfielded image sequences can already be used for scientific investigations but still suffer from residual atmospheric distortions which the adaptive optics system cannot cope with. Sequences recorded with a rate of 10-20 frames per second and a short exposure time $(<50 \mathrm{~ms})$ are post-facto reconstructed employing the Kiepenheuer-Institute Speckle Interferometry Package (KISIP, Wöger \& von der Lühe 2008) to approach the diffraction-limited spatial resolution.

\subsection{Spectrograph camera}

Observation: the spectrograph camera records a onedimensional spectrum of the selected input source (see Sect. 2.1). We use an ANDOR NEWTON CCD camera with a pixel size of $13.5 \mu \mathrm{m}$ and a chip size of $2048 \times 512$ pixels. Owing to the single fiber feed of the spectrograph, the signal is concentrated in only few (typically two to three) adjacent pixel rows of the CCD chip. However, due to effects of instrument internal seeing, or changes in the ambient air pressure and temperature, the spectrum suffers from potential drifts. Detailed characterizations of the spectrograph stability (Doerr 2015) revealed that long-term variations of the environmental conditions can lead to shifts of the LFC spectrum of a few hundred meters per second within several hours. In addition, the short-term variation shows an instrumental jitter at the scale of $10 \mathrm{~s}$ with an amplitude up to a few $\mathrm{m} \mathrm{s}^{-1}$. With the LFC spectra recorded at a cadence of a second or below, we can account for these drifts. To consider spatial shifts of the spectrum across the camera sensor, a region of around twenty rows around the illuminated pixels is read out and binned perpendicular to the dispersion axis. With a dispersion of $19 \mathrm{pm} \mathrm{mm}^{-1}$ at a wavelength of $\lambda=550 \mathrm{~nm}$, the 2048 pixel wide spectrum covers a spectral range of $0.53 \mathrm{~nm}$. The spectral resolution of the spectrograph amounts to about 800000 at that wavelength. A typical observation sequence with LARS consists of the repetition of a two-channel cycle. For solar observations, one cycle constitues of the successive measurement of the frequency comb spectrum and the solar spectrum. An exemplary cycle is shown in Fig. 12. Typical integration times are around $1 \mathrm{~s}$, depending on the solar target. In the case of a Quiet Sun region at disk center and a single exposure for $1 \mathrm{~s}$, a signal-to-noise of 200 is reached for the spectral continuum around $550 \mathrm{~nm}$. At this signal level, dark current and read noise do not play a significant role. As reported in Doerr (2015), the signal level decreases only to a minor fraction when changing between the $10^{\prime \prime}, 3^{\prime \prime}$, and $1^{\prime \prime}$ fiber-coupling units. This is because the coupling efficiency of the multi-mode light source to a single-mode fiber increases with decreasing field of view, in our case by one magnitude from $10^{\prime \prime}$ to $3^{\prime \prime}$, and $3^{\prime \prime}$ to $1^{\prime \prime}$ each.

Given the temporal scales of seconds, very fast camera readout is not important. During the cycle, the fiber switch can change the input channel within $2 \mathrm{~ms}$. This sets a total cycle time of a few seconds. By repeating the cycle, the frequency comb and solar spectra are observed alternately. Therefore the solar spectrum can be calibrated by the interpolation between the preceding and succeeding comb spectrum. The user defines the number of repetitions and, by this, the observation time of the sequence. The fully automated data capture software writes the data sequence as one file in FITS format. The first dimension contains the 2048 pixels, the second dimension successive cycles (or time). The metadata required by the data pipeline is written into the FITS header.

Data calibration: the observed raw data of the spectrograph camera still contain systematic defects like dissimilar pixel sensitivities and dust on the detector, the background readout, and 


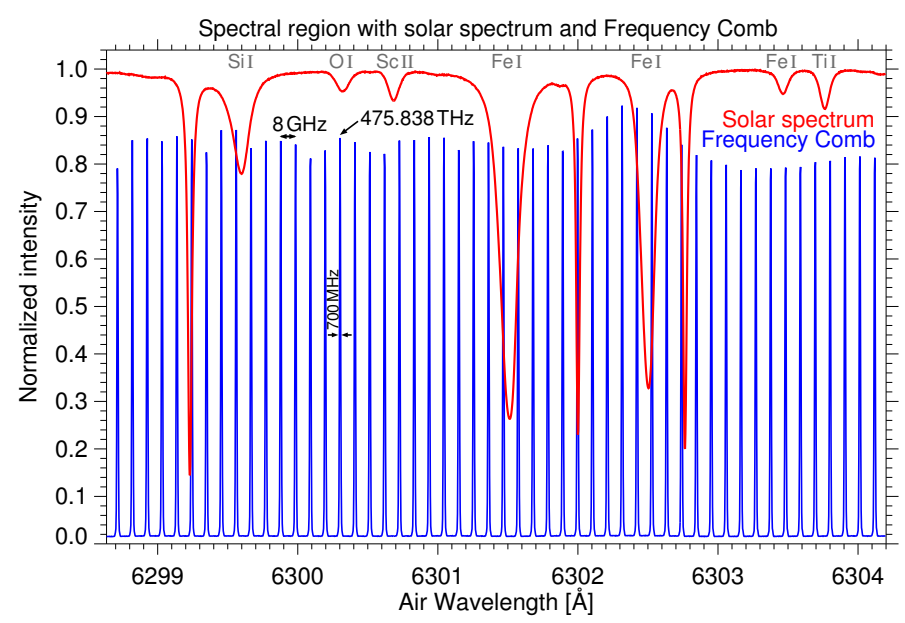

Fig. 12. Spectral region with a typical cycle of solar spectrum and frequency comb signal. The spectrograph camera records a spectral region with a wavelength width of $5.6 \AA$, here centered around $6301.5 \AA$. The solar spectrum in red was normalized to the continuum intensity and consists of several photospheric lines (elements in gray). The three unnamed narrow lines are telluric $\mathrm{O}_{2}$ lines. The frequency comb spectrum is overlaid in blue and consists of 52 equally spaced $(8 \mathrm{GHz})$ emission modes with a full width at half maximum of $700 \mathrm{MHz}$. One frequency mode (here $475.838 \mathrm{THz}$ ) has to be unambiguously identified through its proximity to a spectral line core. The solar and the comb lines are indeed observed sequentially to avoid any deterioration of the solar line profile through the superposition of the comb lines.

large-scale gradients in the spectrograph transmission. A set of "dark" and "flatfield" sequences is recorded to reduce these errors from the spectral sequences. The scheme of the spectral data calibration is shown in Fig. 11 on the left side. Two short dark sequences taken with the covered camera define the readout and background signal of the camera. Since the camera can be cooled to $-90^{\circ} \mathrm{C}$, the dark signal is very low. The exposure times of the darks have to be the same as for the spectral sequence (e.g., "LFC/Sun") and flatfield sequence, respectively. A tungsten lamp emits the continuous flatfield spectrum which contains the instrumental errors when recorded by the camera. The exposure time for the flatfield is adjusted to match the continuum intensity level of the solar spectrum. The raw data (yellow boxes in Fig. 11) are entered in the semi-automated data pipeline. After each dark and flatfield sequence is averaged in time, the gaintable spectrum is calculated as the normalized difference between flatfield and dark. The flatfielded spectral sequence is obtained by subtracting the corresponding dark spectrum from each solar or comb spectrum and dividing by the gaintable spectrum.

In the next processing steps, the solar spectrum has to be calibrated to an absolute wavelength grid (using the comb spectrum) and corrected from inherent systematic wavelength shifts (calculated by an ephemeris code). Given the strong effect of systematic relative motions between the telescope and the light source, the latter substantially depends on the high accuracy of the applied models. The applied ephemeris code developed by Doerr (2015), which in turn is based on NASA's Navigation and Ancillary Information Facility Spacecraft Planet Instrument C-matrix Events (SPICE) toolkit (Acton 1996), computes the relative motion between the observing telescope and the Sun with an accuracy of fractions of $\mathrm{mm} \mathrm{s}^{-1}$. For a ground-based telescope, it includes the orbital motion of the Earth around the Sun, as well as the terrestrial rotation at the location of the observatory, which can add up to a rapidly changing line-of-sight velocity of the order of $\pm 1000 \mathrm{~m} \mathrm{~s}^{-1}$. The next systematic component is the gravitational shift caused by the Sun and Earth according to the General Theory of Relativity which, taken together, amounts to a redshift of $633.3 \mathrm{~m} \mathrm{~s}^{-1}$, everywhere on the solar disk. If desired, the differential rotation of the Sun reaching a line-of-sight velocity of up to $\pm 2000 \mathrm{~m} \mathrm{~s}^{-1}$ at the solar limb can be modeled and reduced for each heliographic position on the solar disk. The ephemerides code calculates the wavelength shifts for each time step of the observed data sequence. The generated file is later applied to the calibrated solar spectrum.

To get the absolute wavelength grid for the solar spectrum, the comb spectrum has to be unambiguously calibrated. The separation of the comb mode is fixed and amounts to $8.0 \mathrm{GHz}$. With this knowledge, each pixel of the detector can be assigned to a fraction of a mode number. To determine the positions of the whole emission modes, each individual comb line is fitted with a Gaussian model with four degrees of freedom (center, width, amplitude, offset). Due to the very good side-mode suppression by the two high-finesse FPCs, the Gaussians fit the comb line center extremely well. The typical statistical error for the measured mode positions is estimated to $5 \mathrm{~cm} \mathrm{~s}^{-1}$ for a single calibration exposure. Only potential asymmetries of the instrumental profile can become a severe systematic issue. In this case, the comb lines shift toward the centroid of the instrumental profile. However, the deviation of the final mode profile from the perfectly symmetrical Gaussian fit is minor, so that this effect is limited to a shift well below $1 \mathrm{~m} \mathrm{~s}^{-1}$ in average. In the next calibration step, the overall pixel-to-mode-number solution is fitted by a polynomial, thus yielding an intermediate frequency solution. For a single measurement, its absolute accuracy was determined to about $60 \mathrm{~cm} \mathrm{~s}^{-1}$. The limiting unmodeled sub-structure in the frequency solution is considered to stem from small $(\sim 50 \mathrm{~nm})$ pixel spacing variations of the CCD detector. Since the repetition rate and offset frequency $(-100 \mathrm{MHz})$ of the comb spectrum are known, it is sufficient to unambiguously identify only one mode with its mode number. We select a well-known solar spectral line and enter its air wavelength. The closest comb mode serves as the reference mode to assign all other modes with a well-defined frequency (e.g., in Fig. 12 the reference mode close to the O I line at $6300.3 \AA$ has a frequency of $475.838 \mathrm{THz}$ ). With this information, all intermediate calibration steps are combined to compute the final frequency (or wavelength) solution. Each individual solar spectrum is then calibrated by the temporal interpolation of the two adjacent LFC calibration functions. To achieve a high spectral accuracy for our measurements, a temporal cadence of only few seconds or less is necessary. In fact, the spectrograph instability introduces the largest error, compared to the line shifts of a few $\mathrm{cm} \mathrm{s}^{-1}$ from the LFC and CCD detector. Instrument internal seeing, that is, a spectrograph jitter at the scale of $10 \mathrm{~s}$, influences the repeatability of the observation. In periods of good instrumental seeing, the error typically is of the order of a few $\mathrm{cm} \mathrm{s}^{-1}$ but can increase up to a few $\mathrm{m} \mathrm{s}^{-1}$. For measurements with $1 \mathrm{~s}$ cadence, this limits the total accuracy to around $1 \mathrm{~m} \mathrm{~s}^{-1}$, or better.

Since the transmission plateau of the spectrograph is quite narrow and the observed spectral band is not always well centered within the diffraction order selected with the predisperser, gradients in the spectral continuum may occur. To correct for this effect, the Fourier Transform Spectrometer (FTS, Neckel 1999) atlas spectrum is overplotted to the LARS observations. The FTS atlas features a spectral resolution of 400000 and an accuracy of around $\pm 50 \mathrm{~m} \mathrm{~s}^{-1}$ in the visible which is sufficient for a comparison of both spectra. By selecting individual continuum positions, a polynomial correction function is fitted and stored. In the last 

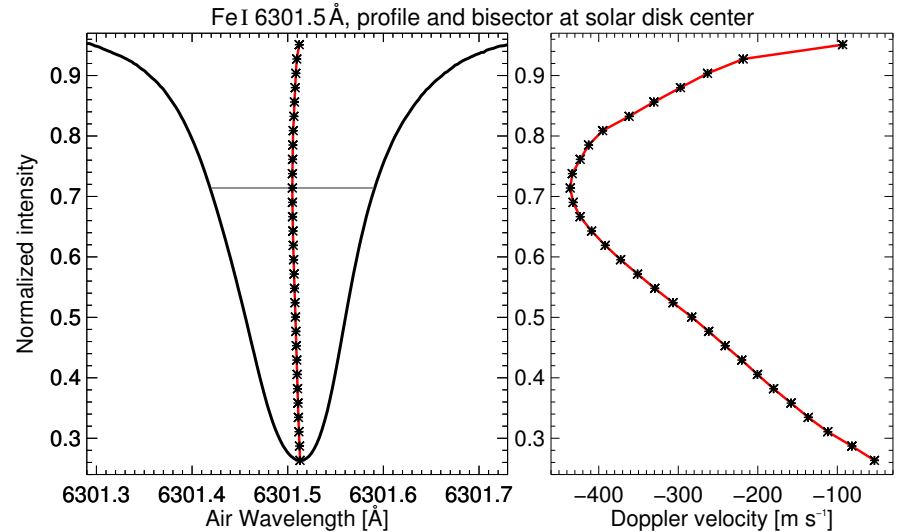

Fig. 13. Spectral line profile and bisectors of Fe I $6301.5 \AA$ observed in the Quiet Sun at disk center. The line profile is drawn as a black solid line in the left panel, with intensities normalized to the spectral continuum and air wavelength in $\AA$. An exemplary bisector is indicated as a gray horizontal line. All center positions of the bisectors are shown as black asterisks, their interpolation is displayed as a red solid line. In the right panel, the wavelengths were translated into Doppler velocities in $\mathrm{m} \mathrm{s}^{-1}$.

step of the data calibration, this correction function and the computed wavelength calibration are applied to the sequence of solar spectra. A final, calibrated LARS spectrum is plotted in Fig. 12 as a red curve.

\section{First results and outlook}

In 2016, three observation campaigns were carried out with the LARS instrument. In total 58 days of telescope time at the VTT were at our disposal to test the instrument with the upgraded LFC and to perform solar observations. All data were calibrated with the processing techniques described in Sect. 3. The main goal was to measure spectral line shifts caused by solar convection and acoustic waves with an unprecedented spectral accuracy. In a series of forthcoming papers, we will present the results for the center-to-limb variation of the convective blueshift observed with several frequently used spectral lines (e.g., the Fe I lines at $6301.5 \AA, 6302.5 \AA, 6173 \AA, 5250 \AA$, and Na I $5896 \AA$ ). In a further article, we will address umbral oscillations measured with photospheric (Ti I $5714 \AA$ ) and chromospheric (Na I $5896 \AA$ ) lines. For now, we want to give a brief outlook on the first results and discuss future prospects with LARS.

With a spectral resolution $(\Delta \lambda / \lambda)$ of $700000-800000$ and a spatial sampling of a $1^{\prime \prime}-10^{\prime \prime}$ wide region, LARS enables advanced spectroscopic investigations of line profiles. In the left panel of Fig. 13, the intensity profile of the Fe I $6301.5 \AA$ line is shown with its normalized intensity plotted against the air wavelength in $\AA$. The data was observed on May 132016 at 08:12 UT with the $10^{\prime \prime}$ fiber-coupling unit in a Quiet Sun region at the solar disk center. The time series of 20 min was wavelength calibrated and temporally averaged. The figure is a magnification of the spectral range also shown in Fig. 12. The line has a depth of more than $70 \%$ and a full width at half maximum of around $0.14 \AA$. To attain detailed information on the line shape and Doppler velocities along the sampled atmospheric layers, a bisector analysis was performed on the profile. The center wavelengths are computed for 30 equidistant height levels starting from the line minimum up to $96 \%$ of the continuum intensity. The single positions are marked as black asterisks in Fig. 13. The interpolated curve was added as a solid red line and highlights the asymmetric

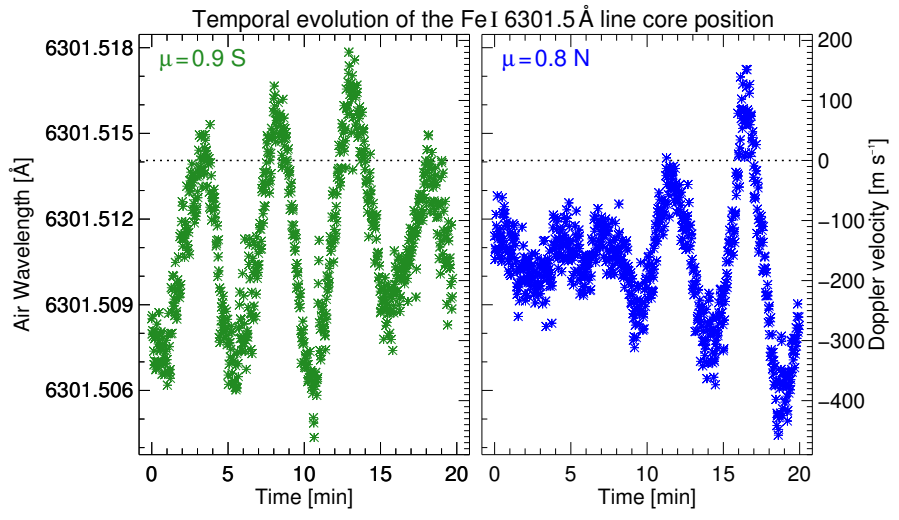

Fig. 14. Temporal variation of the observed Fe I $6301.5 \AA$ line core position. The oscillatory evolution is displayed for two 20 min-sequences observed in the Quiet Sun. Left panel: measured on October 142016 at 09:34 UT at the heliocentric parameter $\mu=0.9$ on the southern axis of the solar meridian. Right panel: october 132016 at 15:07 UT at $\mu=0.8$ on the northern axis of the meridian. The single measurements (asterisks) have a cadence of $1.5 \mathrm{~s}$. The left $y$-axes scale the wavelength in $\AA$, the right $y$-axes are the translated Doppler velocities in $\mathrm{m} \mathrm{s}^{-1}$.

shape of the line profile. To convert the shifts from air wavelength to Doppler velocities, the exact reference position is required. We typically take the observed air wavelength from the atomic spectra database of the National Institute of Standards and Technology (NIST, Kramida et al. 2015). For several spectral lines the reference wavelength positions can be measured with the hollow-cathode lamps available with LARS. The reference wavelength is subtracted from the observed wavelength and the remaining difference is translated $(\Delta \lambda / \lambda=\Delta v / c)$ into a Doppler shift. The subtraction of the gravitational redshift of $633 \mathrm{~m} \mathrm{~s}^{-1}$ (uniform for the solar disk) yields the correct line-ofsight velocities in the solar atmosphere. Thanks to the absolute wavelength calibration, there is no need to refer to some (arbitrarily chosen) area of Quiet Sun, or the like. At the solar disk center, a redshift is really a downflow, and a blueshift is an upflow in our case. This is limited by the accuracy of the reference wavelength, which is better than $\pm 1 \mathrm{~m} \AA$ (NIST) for the selected well-studied lines. The right panel in Fig. 13 reflects the bisector analysis from the left panel, plotted against Doppler velocities in $\mathrm{m} \mathrm{s}^{-1}$.

The differential shifts leading to the " $\mathrm{C}$ "-shaped bisector curve originate from the physical conditions in the solar atmosphere (Balthasar 1984). The upflowing material in the centers of granules is hotter than the downward moving gas in intergranular lanes, hence the upflows contribute more to the spatially averaged line profiles, causing a net blueshift of the line. Since the convective flow speed decreases with atmospheric height, the negative (upward) velocities decrease toward the line core. In the case of the Fe I $6301.5 \AA$ line, the velocity declines from around $-440 \mathrm{~m} \mathrm{~s}^{-1}$ at a normalized intensity of 0.7 to around $-50 \mathrm{~m} \mathrm{~s}^{-1}$ at the line minimum. Beyond this example, the high spectral resolution, wavelength accuracy, and fast temporal cadence of LARS observations enable the detection of subtle and fast changes in the physical properties of the solar atmosphere.

The investigation of acoustic waves in the solar atmosphere with LARS could become invaluable for the research field of local Helioseismology. Magneto-acoustic waves in sunspots and pores can be observed with the highest spectral accuracy. Precise measurements of absolute $p$-mode oscillations can be performed in a confined region of the Sun. Two examples of $p$-mode observations in Quiet Sun regions are presented in Fig. 14. The panels 
show the temporal evolution of the line center of Fe I $6301.5 \AA$ in air wavelength (left $y$-axes) and corresponding Doppler velocities (right axes). In this case, the line center was defined as the minimum position of a parabolic fit to the inner line core $( \pm 20 \mathrm{~m} \AA)$. The time sequences were performed with a cadence of $1.5 \mathrm{~s}$ and took $20 \mathrm{~min}$. Both sequences were measured with the $10^{\prime \prime}$ fiber-coupling unit. The left time series (green asterisks) was recorded on the southern axis of the solar meridian at an heliocentric angle of $\alpha=26^{\circ}$ between the line of sight and the local normal to the solar surface. The associated heliocentric parameter is $\mu=\cos \alpha=0.9$. The right time series (blue asterisks) was observed at an heliocentric angle $\alpha=37^{\circ}$, or $\mu=0.8$, on the northern meridional axis. The convective blueshift sets the velocities by $150 \mathrm{~m} \mathrm{~s}^{-1}$ to negative values. $P$-mode oscillations with a period of $5 \mathrm{~min}$ and amplitude of up to $300 \mathrm{~m} \mathrm{~s}^{-1}$ are clearly recognizable in both examples. In addition, a destructive interference of $p$-mode waves is apparent in the first half of the right series.

Three-dimensional hydrodynamics and magnetohydrodynamics simulations of the quiet solar atmosphere have been claimed to be "realistic" (Pereira et al. 2013) or "highly realistic" (Scott et al. 2015), and have been used to calibrate the center-to-limb variation of the convective blueshift (de la Cruz Rodríguez et al. 2011). LARS provides measurements of unprecedented quality that will allow for a detailed comparison of a number of spectral lines. We will measure the center-to-limb variation of the convective blueshift, and of the corresponding line asymmetries (C-shapes). Since these line properties are intimately coupled with the temperature and velocity gradients in the solar atmosphere, the center-to-limb variations of these quantities will give tight constraints for realistic numerical models of the solar atmosphere. As models of the solar atmosphere are fundamental for the interpretation of stellar spectra, such measurements can also have a great impact on our understanding of stellar atmospheres. Convective blueshifts on stars other than the Sun are a source of significant systematic errors for the determination of correct radial velocities. Realistic models of stellar atmospheres must faithfully reproduce the observed spectral lines and their center-to-limb variation in the solar atmosphere, before they can be applied to stellar atmospheres.

The observed temperature of sunspot umbrae suggests the presence of convection, which would also produce some (weak) convective blueshift. To investigate this topic, we intend to determine the absolute wavelength of suitable spectral lines in sunspot umbrae to derive the combined effect of gravitational redshift and convective blueshift. The different center-to-limb behavior of both effects will allow us to distinguish between the two contributions. The main limitation for this investigation may come from the rapidly approaching minimum of the current solar activity cycle.

Using the improved possibilities for spectral calibration with laser frequency combs and Fabry-Pérot interferometers, new solar flux atlases have been produced. The atlases of Molaro et al. (2013) using the High Accuracy Radial velocity Planet Searcher (HARPS) and of Reiners et al. (2016) using the Fourier transform spectrograph at the Institute for Astrophysics Göttingen provide a wavelength accuracy on the order of $\pm 10 \mathrm{~m} \mathrm{~s}^{-1}$, thus exceeding the accuracy of the renowned FTS atlases of Kurucz et al. (1984), Neckel (1999), or Wallace et al. (2011) by one order of magnitude. With LARS, we could construct a diskcenter spectral atlas as well. But since the narrow spectral window of the LARS spectrograph does not permit a large wavelength coverage, the fragmented measurement would be very time-consuming and laborious. For mutual benefit, we will provide accurate wavelengths and asymmetries for selected lines of high astrophysical interest to which future studies and atlases can refer.

Acknowledgements. We thank all colleagues at the Kiepenheuer-Institut für Sonnenphysik, at Menlo Systems, and at the Max-Planck-Institut für Quantenoptik who worked on the development of the instrument, and the upgrade of the laser frequency comb in 2016. We especially thank Thomas Sonner, as well as Roberto Simoes, Frank Heidecke, Andreas Fischer, and Oliver Wiloth for their help in realizing the upgrade of the solar fiber feed optics in 2017. The development of the LARS instrument and the operation of the Vacuum Tower Telescope at the Observatorio del Teide on Tenerife were performed by the KiepenheuerInstitut für Sonnenphysik, which is a public law foundation of the State of BadenWürttemberg. This work is part of a Post-Doc project funded by the Deutsche Forschungsgemeinschaft (DFG, Ref.-No. Schm-1168/10). The initial astro-comb project for the VTT was funded by the Leibniz-Gemeinschaft through the "Pakt für Forschung und Innovation". Finally, we would like to thank Reiner Volkmer for his comments on the manuscript.

\section{References}

Acton, C. H. 1996, Planet. Space Sci., 44, 65

Balthasar, H. 1984, Sol. Phys., 93, 219

Beckers, J. M. 1977, ApJ, 213, 900

Collados, M., López, R., Páez, E., et al. 2012, Astron. Nachr., 333, 872

de la Cruz Rodríguez, J., Kiselman, D., \& Carlsson, M. 2011, A\&A, 528, A113

Doerr, H.-P. 2015, Ph.D. Thesis, University of Freiburg

Doerr, H.-P., Kentischer, T. J., Steinmetz, T., et al. 2012a, in SPIE Conf. Ser., 8450

Doerr, H.-P., Steinmetz, T., Holzwarth, R., Kentischer, T., \& Schmidt, W. 2012b, Sol. Phys., 59

Edlén, B. 1953, J. Opt. Soc. Am., 43, 339

Edlén, B. 1966, Metrologia, 2, 71

Kentischer, T. J., Bethge, C., Elmore, D. F., et al. 2008, in Ground-based and Airborne Instrumentation for Astronomy II, Proc. SPIE, 7014, 701413

Kramida, A., Yu. Ralchenko, Reader, J., \& and NIST ASD Team 2015, NIST Atomic Spectra Database (ver. 5.3), http://physics.nist.gov/asd

Kurucz, R. L., Furenlid, I., Brault, J., \& Testerman, L. 1984, Solar flux atlas from 296 to $1300 \mathrm{~nm}$ (National Solar Observatory)

Lovis, C., \& Pepe, F. 2007, A\&A, 468, 1115

Molaro, P., Esposito, M., Monai, S., et al. 2013, A\&A, 560, A61

Neckel, H. 1999, Sol. Phys., 184, 421

Pereira, T. M. D., Asplund, M., Collet, R., et al. 2013, A\&A, 554, A118

Probst, R. A., Wang, L., Doerr, H.-P., et al. 2015, New J. Phys., 17, 023048

Reiners, A., Banyal, R. K., \& Ulbrich, R. G. 2014, A\&A, 569, A77

Reiners, A., Mrotzek, N., Lemke, U., Hinrichs, J., \& Reinsch, K. 2016, A\&A, 587, A65

Schmidt, W., von der Lühe, O., Volkmer, R., et al. 2012, Astron. Nachr., 333, 796

Schroeter, E. H., Soltau, D., \& Wiehr, E. 1985, Vistas Astron., 28, 519

Scott, P., Asplund, M., Grevesse, N., Bergemann, M., \& Sauval, A. J. 2015, A\&A, 573, A26

Steinmetz, T., Wilken, T., Araujo-Hauck, C., et al. 2008, Science, 321, 1335

Wallace, L., Hinkle, K. H., Livingston, W. C., \& Davis, S. P. 2011, ApJS, 195, 6

Wilken, T., Curto, G. L., Probst, R. A., et al. 2012, Nature, 485, 611

Wöger, F., \& von der Lühe, II, O. 2008, in SPIE Conf. Ser., 7019, 1 